\title{
Special issue on challenges in emerging environmental contaminants CEEC19
}

\author{
Giovanni Libralato $^{1} \cdot$ Rosa Freitas $^{2} \cdot$ Isabella Buttino ${ }^{3} \cdot$ Augustine Arukwe $^{4} \cdot$ Camilla Della Torre $^{5}$ \\ Published online: 17 June 2020 \\ (C) Springer-Verlag GmbH Germany, part of Springer Nature 2020
}

Contamination of natural ecosystems by man-made chemical pollutants is considered one of the main drivers of global changes. The widespread and persistent input of pollutants into the environment is so large that their impact on wildlife can outpace other global stressors. While the remediation, mitigation, and minimization of legacy pollutants is currently a challenging task, new substances are appearing as contaminants of emerging concern (CECs). These include, but not limited to-rare earth elements, micro- and nano-plastics, nanomaterials, perfluoroalkyl and polyfluroalkyl substances (PFASs), pharmaceuticals and personal care products (PPCPs), illicit drugs, flame retardants, artificial sweeteners, and ionic liquids. Most of these compounds are released into wastewater and are only partially removed by wastewater treatment plants. Thus, they can be discharged into surface water bodies in their native or metabolized form, generating potential adverse effects to the biota, and compromising water resources quality including drinking water supplies. Indeed, many of these contaminants can still exert toxic effects at very low concentrations (ng/L or even lower) and their environmental occurrence might still be hardly detectable. This is even more challenging for physical contaminants at the micro- and nanoscale. The past experience

Responsible Editor: Philippe Garrigues

Camilla Della Torre

Camilla.dellatorre@unimi.it

1 Department of Biology, University of Naples Federico II, Naples, Italy

2 Department of Biology \& CESAM, University of Aveiro, Aveiro, Portugal

3 Section of ecological risk assessment in marine coastal areas, Italian Institute for Environmental Protection and Research, Livorno, Italy

4 Department of Biology, Norwegian University of Science and Technology (NTNU), Trondheim, Norway

5 Department of Bioscience, University of Milan, Milan, Italy with priority man-made pollutants — such as DDT — highlights the need for the scientific community to foster a proactive approach towards CECs, in order to prevent and minimize their long-term adverse impacts on the natural ecosystems. Nevertheless, for several CECs, the implementation of effective management strategies is hindered by the gaps of knowledge and technical limitations. Indeed, the actual environmental levels of many CECs are lacking and for some contaminants measurement technologies are still not adequate and analytical procedures not standardized. Moreover, the processes and interactions with biological systems that drive toxic effects are still poorly understood and available data are sometimes contradictory. Furthermore, even if in natural conditions organisms are exposed to complex mixture of contaminants, information on the impacts of combination of pollutants is still very scarce.

It became also evident that toxicity tests developed for priority pollutants often fail to adequately address their potential adverse effects due to their unique physicochemical properties. In this view, the identification of target species representative of all environmental compartments (air, water, soil, sediment) and different ecosystems, including remote areas, is important in developing adequate and comprehensive risk assessment tools.

Within this scenario, the SI focused on the main challenges about CECs, merging different approaches and points of view, and includes research papers that link environmental quality implications to ecosystem health.

Thus, the current SI contains 11 papers dealing with different classes of CECs including pesticides, surfactants, nanoparticles and microplastics, tested on various environmental compartments and matrices and addressing different issues, such as the following:

- Technical challenges for identification and quantification of emerging contaminants in environmental matrices and biota

- New researches on the mechanisms that underlie the toxicity of emerging contaminants 
- Novel ecotoxicological approaches to assess the ecotoxicity of emerging environmental contaminants

- Standardization of protocols and environmental policy proposals

In detail:

Tasselli and Guzzella (2020) developed analytical methods for the measurement of polycyclic musk fragrances (PMFs) in wastewater treatment plants (WWTPs). Specifically, for wastewater solid phase extraction followed by quantification in GC-MS/MS were employed, while ultrasonic bath extraction and GC-Ion trap quantification were used for activated sludges. The methods were also tested on an Italian WWTP and results highlighted the concern related to the release of PMFs in the environment from WWTPs, being current technologies for sewage treatment poorly efficient in the removal of such pollutants.

Adeogun et al. (2020) reported a first screening on the occurrence of microplastics (MPs) in several edible fish from a municipal water supply in Nigeria. MPs were present in the stomach of $70 \%$ of analyzed individuals. Authors also highlighted that MPs uptake is strongly influenced by ecological features such as trophic level and feeding habits. The highest incidence of MPs was observed in benthopelagic primary consumer species, while pelagic species at higher trophic levels ingested less MPs.

Prato et al. (2019) assessed for the first time the chronic toxicity of $\mathrm{ZnO}$ nanoparticles ( $\mathrm{ZnONPs}$ ) on the marine copepod Tigriopus fulvus. Authors showed that several reproductive traits were affected by $\mathrm{ZnONPs}$ at environmental concentrations, such as brood duration, brood size, and brood number, and highlighted that fecundity and egg viability could be considered as sensitive endpoints of chronic toxicity for this pollutant.

The paper by Beegam et al. (2019) showed another harmful effect of $\mathrm{ZnO}$ particles (1-1.2 and $1.4 \mu \mathrm{m})$ such as the ability to trigger morphological alterations in relevant organs of juvenile seabream Sparus aurata. The histopathological approach showed that gills, muscle, and liver were targeted by $\mathrm{ZnO}$ particles, and the effects were size-dependent.

Socoowski Britto et al. (2019) investigated a relevant, but still controversial aspect of NPs toxicity, such as the interaction with other pollutants. Specifically, the combined effects of graphene oxide and copper were assessed in the manila clam Ruditapes philippinarum. The authors assessed also the combined toxicity under different $\mathrm{pH}$ levels, representative of actual and predicted ocean acidification scenarios, highlighting that this environmental parameter greatly influences the ecotoxicity of nanoparticles.

Koagouw and Ciocan (2019) investigated the adverse effects of the non-steroidal anti-inflammatory drug paracetamol on the blue mussels Mytilus edulis. Exposure to environmental concentration of paracetamol elicited histological alterations in gonad tissue as hemocytic infiltration and follicle degradation. The pharmaceutical induced also the misregulation of genes involved in reproduction (VTG,V9, $E R 2)$ and apoptosis (CASP8, BCL2, FAS). The genes investigated in this study emerged as biomarker candidates for the assessment of the impacts of pharmaceuticals in mussels.

Di Nica et al. (2019) demonstrated that the pesticide chlorpyrifos is an emerging contaminants also in the pristine Alpine environment at high latitudes, affecting swimming behavior of the chironomid Diamesa zernyi larvae at concentrations closed to those found in the surface freshwater streams. The reduction of locomotor capability could be due to a reallocation of energetic reserves to combat oxidative stress induced by low concentrations of the pesticide. Swimming behavior in larvae is a relevant ecological trait and any variation will result to long-term negative impacts in the whole community.

Pescatore et al. (2020) assessed the persistence of the organophosphate insecticide chlorpyrifos (CPF) and the anionic surfactant sodium lauryl ether sulfate (SLES) on soil and their impacts on natural microbial community. Authors tested in microcosm the contaminants alone and in combination. The presence of SLES modified the microbial degradation of CFP. The approach showed also synergistic effects of CPF and SLES on bacterial activity and composition.

Bellino et al. (2019) studied the potential criticalities in environmental monitoring and management, looking at spatial outliers (SO). Authors used two different approaches for the $\mathrm{SO}$ detection in river ecosystems related to several sets of parameters (organic $\mathrm{C}$, inorganic $\mathrm{C}, \mathrm{Ca}, \mathrm{Co}, \mathrm{Cr}, \mathrm{Fe}, \mathrm{K}, \mathrm{Mg}$, $\mathrm{Mn}, \mathrm{N}, \mathrm{Na}, \mathrm{P}, \mathrm{S}, \mathrm{Si}, \mathrm{V}, \mathrm{Zn}, \mathrm{Cl}^{-}, \mathrm{F}^{-}, \mathrm{NO}_{3}{ }^{-}, \mathrm{SO}_{4}{ }^{2-}$, chlorophyll $\mathrm{a}$, chlorophyll $\mathrm{b}$, pheophytin a, pheophytin $\mathrm{b}$, total carotenoids, $\mathrm{pH}$, and electrical conductivity), including emerging contaminants. Criticality scenarios were derived, highlighting both the strengths and the limitations of the proposed approaches especially in relation to the classic threshold-based methods.

A detailed literature review regarding the occurrence of illicit drugs and of their biological effects on aquatic ecosystems, by Fontes et al. (2020), is also reported in this SI. Data, referred to the major groups of illicit drugs including in the categories of hallucinogens, stimulants, or psychoactive drugs, include studies concerning their concentrations in different aquatic matrices and ecotoxicological effects on nontarget aquatic organisms. Drug concentrations found in seafood represent a concrete risk for human health. The review highlights on the inadequacy of sewage treatment systems in the removal of illicit drugs, especially in developing countries, the need to monitor, with standardized protocols, these compounds, and the importance to include such monitoring programs in environmental legislation.

Manfra et al. (2019) discussed the policies to authorize the use of dispersants to mitigate the contamination by petroleum hydrocarbons in case of accidental marine oil spills evidencing the need for harmonizing their approval procedures at 
European level. The comparison of French and Italian requirements suggested a tiered approach addressing dispersants ecotoxicological assessment. The proposed guideline included two mandatory toxicity tests (i.e., algal growth inhibition test and crustacean mortality test) potentially joint to a fish mortality test, whenever necessary, in accordance to animal welfare.

Guest Editors are grateful to the anonymous reviewers who contributed with their comments and suggestions to improve the overall quality of papers of CEEC 19.

\section{References}

Adeogun AO, Ibor OR, Khan EA, Chukwuka AV, Omogbemi ED, Arukwe A (2020) Detection and occurrence of microplastics in the stomach of commercial fish species from a municipal water supply lake in southwestern Nigeria. Environ Sci Pollut Res. https://doi.org/ 10.1007/s11356-020-09031-5

Beegam A, Lopes M, Fernandes T, Jose J, Barreto A, Oliveira M, Soares AMVM, Trinidade T, Sabu T, Pereira M (2019) Multiorgan histopathological changes in the juvenile seabream Sparus aurata as a biomarker for zinc oxide particles toxicity. Environ Sci Pollut Res. https://doi.org/10.1007/s11356-019-05949-7

Bellino A, Alfani A, De Riso L, Baldantoni D (2019) Multivariate spatial analysis for the identification of criticalities and of the subtended causes in river ecosystems. Environ Sci Pollut Res. https://doi.org/ 10.1007/s11356-019-07198-0

Di Nica V, Muñiz González AB, Lencioni V, Villa S (2019) Behavioural and biochemical alterations by chlorpyrifos in aquatic insects: an emerging environmental concern for pristine Alpine habitats. Environ Sci Pollut Res. https://doi.org/10.1007/s11356-01906467-2

Fontes MK, Maranho LA, Seabra Pereira CD (2020) Review on the occurrence and biological effects of illicit drugs in aquatic ecosystems. Environ Sci Pollut Res. https://doi.org/10.1007/s11356-02008375-2

Koagouw W, Ciocan C (2019) Effects of short-term exposure of paracetamol in the gonads of blue mussels Mytilus edulis. Environ Sci Pollut Res. https://doi.org/10.1007/s11356-019-06861-w

Manfra L, Tornambè A, Guyomarch J, Duboscq K, Faraponova O, Sebbio C (2019) Could a harmonized tiered approach assess dispersant toxicity in Italy and France? Environ Sci Pollut Res. https://doi. org/10.1007/s11356-019-06535-7

Pescatore T, Patrolecco L, Rolando L, Spataro F, Rauseo J, Grenni P, Ademollo N, Barra Caracciolo A (2020) Co-presence of the anionic surfactant sodium lauryl ether sulphate and the pesticide chlorpyrifos and effects on a natural soil microbial community. Environ Sci Pollut Res. https://doi.org/10.1007/s11356-020-08840-y

Prato E, Parlapiano I, Biandolino F, Rotini A, Manfra L, Berducci MT, Maggi $\mathrm{C}$ et al (2019) Chronic sublethal effects of $\mathrm{ZnO}$ nanoparticles on Tigriopus fulvus (Copepoda, Harpacticoida). Environ Sci Pollut Res. https://doi.org/10.1007/s11356-019-07006-9

Socoowski Britto R, Nascimento JP, Serode T, Santos AP, Soares AMVM, Figueira E, Furtado C et al (2019) The effects of coexposure of graphene oxide and copper under different $\mathrm{pH}$ conditions in Manila clam Ruditapes philippinarum. Environ Sci Pollut Res. https://doi.org/10.1007/s11356-019-06643-4
Tasselli S, Guzzella L Polycyclic musk fragrances (PMFs) in wastewater and activated sludge: analytical protocol and application to a real case study

Publisher's note Springer Nature remains neutral with regard to jurisdictional claims in published maps and institutional affiliations.

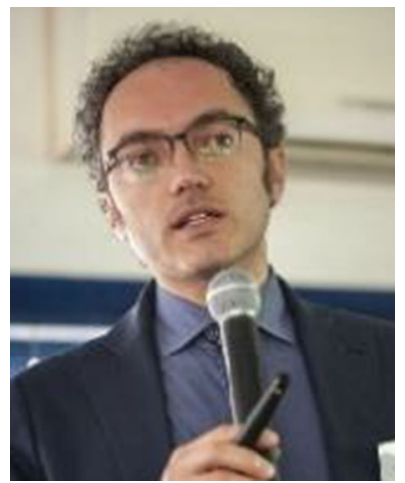

Giovanni Libralato is an associate professor at the University of Naples Federico II (Italy). He was visiting researcher and visiting professor at Université de Nice Sophia-Antipolis (2008) and Université de Lorraine (20132015). He teaches Hygiene and laboratory, Methods in Epidemiology, Hygiene and risk management and General and Applied Hygiene. His main research interests are related to applied environmental hygiene including aquatic (eco)toxicology, nano(eco)toxicology, toxicant effects on populations, factors modifying toxicity, inorganic water chemistry, wastewater treatment, soil and sediment remediation, recovery and reuse of by-products, and water reuse. His main research products can be found in SCOPUS (ID 14825276200), ORCID (0000-0001-8507-900X), RESEARCHGATE and Google Scholar (profile: Giovanni Libralato). email: giovanni.libralato@unina.it

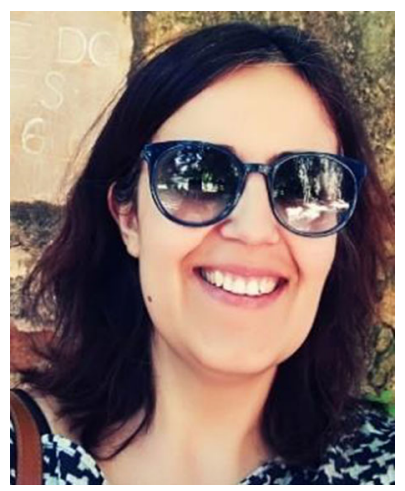

Rosa Freitas is a researcher at the University of Aveiro (UA). She has been investigating the performance of marine biological resources under a changing environment, assessing the toxicity of different stressors, namely contaminants (including pharmaceuticals, carbon nanoparticles, and e-waste elements) and climate change (CC)-related factors. As a result of her research, she has published more 160 papers, presented more than 80 oral communications in national and international meetings. She is frequently invited to international panels for projects and grant evaluation, being also reviewer of several ISI journals. She currently leads a team of more than 20 members, including post-docs, $\mathrm{PhD}$ and MSc students, and grant holders, creating a network with international institutions. Rosa Freitas has also been committed to attract funding to UA, being PI or member at the UA of several national and international projects. At the Biology Dep. (UA), she has been teaching at MSc and BSc programs since 2010. email: rosafreitas@ua.pt 


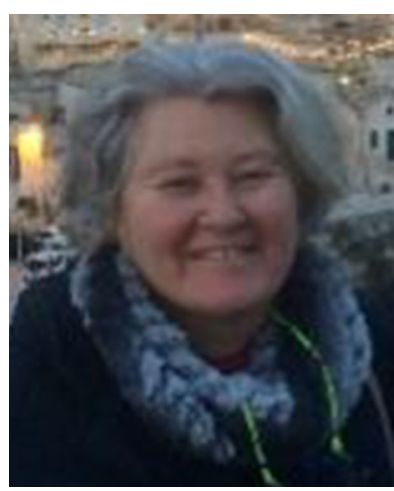

Isabella Buttino is a marine biologist at the Italian Institute for Environmental Protection and Research (ISPRA) - Ecological Risk Assessment in Marine Coastal Areas Section. She promoted the development of Plankton Biology Laboratory and contributed to the knowledge of the physiology and ecotoxicology of marine zooplanktonic organisms, in particular copepods. The main interest is to understand the effects of contaminants on the development and reproduction of marine zooplankton organisms, which in turn can indirectly impact the whole productivity at sea. Recently, she has focused her research on the effects induced by emerging contaminants (such as nanoparticles) on zooplankton (copepods and sea urchin larvae) both at toxico-genomic and physiological level, to investigate in depth the perturbations induced by chemicals at the level of species up to the population, in marine environment. She has more than 60 peer-reviewed publications and she is editor in 6 thematic books, including guidelines for ecotoxicological protocols dedicated to the Environmental Agencies. She is guest professor at the Zhejiang Ocean University (PRC) and has been reviewer for many $\mathrm{PhD}$ and Master thesis in Italy, France, and PRC. email: isabella.buttino@isprambiente.it

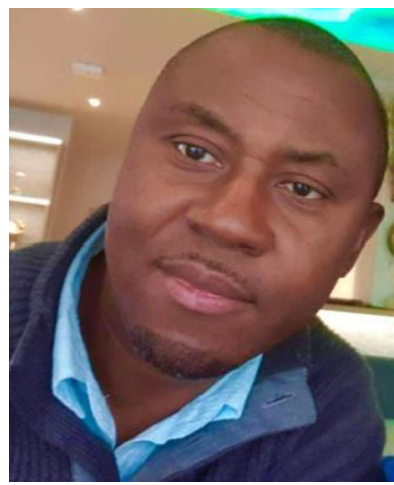

Augustine Arukwe is a professor of molecular toxicology at the Department of Biology, Norwegian University of Science and Technology (NTNU) Trondheim, and European registered toxicologist. His experience and research have addressed and still address issues of impact/risk assessment, the functional and regulatory aspects of differentially expressed genes, proteins and enzymes, and the general stress of contaminants, including endocrine modulators in wildlife species and fish reproductive physiology. He has worked in the USA and Canada under different academic positions. He was a member of the Scientific Steering Committee and panel member on Contaminants for Norwegian Scientific Committee for Food Safety. Arukwe's major research focus has been on the development and validation of sensitive biomarkers of ecotoxicology, endocrine, and chemical exposure in organisms (fish, marine birds, amphibians, and marine mammals). He has developed teaching and research tools into the molecular basis and mechanisms of toxicity and physiological responses. He is also extensively involved in several capacity building projects in Nigeria, Tanzania, South Africa and Vietnam. http://orcid.org/0000-0002-2134-4799. email: augustine.arukwe@ntnu.no

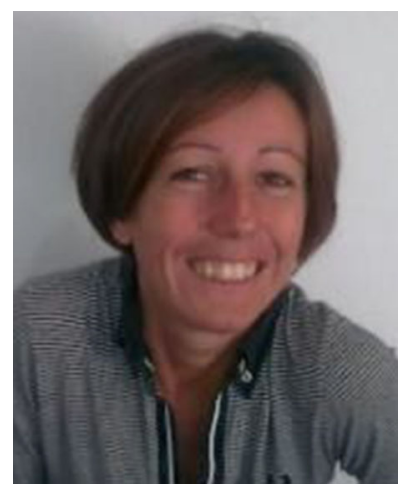

Camilla Della Torre is currently an assistant professor in Ecology at the Department of Biosciences, University of Milan. Her research focuses on the ecotoxicity of emerging pollutants, and in particular nanomaterials in aquatic models. From 2008 to 2014 she had a Post-doc position at the Department of Physical Earth and Environmental Sciences of the University of Siena working on the application of in vivo and in vitro models for testing the toxicity of legacy and emerging con-

taminants on aquatic ecosystems. She received her $\mathrm{PhD}$ in Environmental Sciences at the Environmental Sciences Department of the Siena University. From 2001 to 2005, she worked with a contract of employment at Central Institute for Marine Research in Rome. Her main activities were the analysis of biochemical biomarkers in fish. 